\title{
Breast Sarcoma
}

National Cancer Institute

\section{Source}

National Cancer Institute. Breast Sarcoma. NCI Thesaurus. Code C4670.

A malignant mesenchymal neoplasm that arises from the breast. Representative examples include angiosarcoma, liposarcoma, leiomyosarcoma, rhabdomyosarcoma, and extraskeletal osteosarcoma. 